\title{
Activator protein 1 (AP-1) contributes to EpCAM- dependent breast cancer invasion
}

\author{
Narendra V Sankpal ${ }^{1}$, John D Mayfield ${ }^{1}$, Mike W Willman ${ }^{1}$, Timothy P Fleming ${ }^{1,2}$ and William E Gillanders ${ }^{1,2^{*}}$
}

\begin{abstract}
Introduction: EpCAM is a cell-surface glycoprotein that is overexpressed in the majority of epithelial carcinomas. However, the functional role of EpCAM in regulating cancer invasion remains controversial, and the mechanism(s) underlying EpCAM-mediated regulation of breast cancer invasion remain to be defined.

Methods: EpCAM expression was manipulated in breast cancer cell lines using RNA interference and cDNA expression constructs. Recombinant EpCAM was used to rescue EpCAM signaling following specific ablation of EpCAM. Protein and gene expression, invasion, transcription factor activity, and protein phosphorylation were measured using standard molecular biology techniques.
\end{abstract}

Results: In loss-of-function, and gain-of-function experiments we demonstrate that EpCAM expression is associated with increased breast cancer invasion in vitro and in vivo. We demonstrate further that specific ablation of EpCAM expression is associated with decreased activator protein-1 (AP-1) transcription factor activity. Phosphoprotein analyses confirm that specific ablation of EpCAM is associated with decreased phosphorylation of the AP-1 subunit c-Jun. Recombinant soluble extracellular EpCAM (rEpCAM) is able to rescue invasion, AP-1 transcription factor activity, and c-Jun phosphorylation in a dose-dependent fashion. Pharmacologic inhibitors, and constitutively active constructs of the c-Jun N-terminal kinase (JNK) signal transduction pathway, suggest that the impact of EpCAM expression on AP-1 transcription factor activity is mediated through the JNK pathway. In functional rescue experiments, forced expression of c-Jun rescues invasion in breast cancer cells following specific ablation of EPCAM.

Conclusions: These data demonstrate for the first time that EpCAM expression can influence the JNK/AP-1 signal transduction pathway, and suggest that modulation of AP-1 transcription factor activity contributes to EpCAMdependent breast cancer invasion. These data have important implications for the design and application of molecular therapies targeting EpCAM.

\section{Introduction}

The epithelial cell adhesion molecule (EpCAM) is a type I transmembrane protein that is localized to the basolateral membrane in the majority of normal epithelial tissues [1]. The functional role of EpCAM in cell adhesion was the focus of early studies, and EpCAM has been demonstrated to be a calcium-independent homophilic cell adhesion molecule [2]. Recent studies have also demonstrated a role for EpCAM in cell signaling, proliferation and invasion [3-7]. EpCAM is perhaps best known for the fact that it is overexpressed in the

\footnotetext{
* Correspondence: gillandersw@wustl.edu

'Department of Surgery, Washington University School of Medicine, Campus Box 8109, 660 South Euclid Avenue, St. Louis, Missouri, 63110, USA Full list of author information is available at the end of the article
}

majority of human epithelial cancers including colorectal, breast, gastric, prostate, ovarian, and lung cancers $[8,9]$. EpCAM was the first human tumor-associated antigen to be identified with monoclonal antibodies [10], and was the first target of monoclonal antibody therapy in humans [11]. Although initial results have been disappointing, a number of second-generation molecular therapies are currently under development [12-17]. Despite this intense interest in EpCAM as a target for molecular therapy, there have been limited attempts to define the functional role of EpCAM in cancer biology.

EpCAM expression in primary cancer specimens has been studied extensively, and a number of studies in the surgical pathology literature have evaluated the

\section{Biomed Central}


association between EpCAM expression and prognosis. One inconsistency in the literature is that EpCAM expression in primary cancer specimens appears to be associated with a favorable prognosis in some cancer types, and an unfavorable prognosis in other cancer types. For instance, EpCAM expression in primary breast cancers appears to be associated with decreased patient survival $[8,18-20]$. However, EpCAM expression in colorectal cancer appears to be associated with improved patient survival [21]. Additional studies in other cancer types have suggested an association with improved patient survival in esophageal cancer [22], gastric cancer [23], and renal cell carcinoma [24,25], and an association with decreased patient survival in ovarian cancer [26], gall bladder cancer [27], and pancreatic cancer [28]. Although these studies are far from definitive, taken together, they do suggest a cancer type-specific role for EpCAM in cancer biology and invasion. This inconsistency is paralleled in functional studies of EpCAM biology performed in vitro. Loss-of-function analyses using RNA interference suggest that EpCAM expression is associated with increased invasion in breast cancer [4], and gain-of-function analyses in colorectal and lung cancers suggest that EpCAM expression is associated with decreased cancer invasion in these cancer types $[29,30]$. A better understanding of the relation between EpCAM and cancer invasion will clearly facilitate the rational design, and successful application of molecular therapies targeting EpCAM in epithelial carcinomas.

In this study we confirm that EpCAM expression is associated with increased breast cancer invasion in vitro and in vivo. In mechanistic studies, we demonstrate for the first time that EpCAM expression can modulate the c-Jun N-terminal kinase (JNK)/activator protein 1 (AP1) signal transduction pathway and target genes. These observations provide important insights into the downstream mediators of EpCAM signaling, and the impact of EpCAM expression on breast cancer invasion and prognosis.

\section{Materials and methods \\ Cell culture}

The MDA-231 and MCF-7 breast cancer cell lines were obtained from the American Type Culture Collection (ATCC, Rockville, MD, USA). The CA1a breast cancer cell line was obtained from Dr. Fred Miller at Wayne State University (Detroit, MI, USA).

\section{RNA interference and lentivirus generation}

The lentiviral construct pSicoR and related expression vectors were obtained from Dr. Tyler Jacks (Massachusetts Institute of Technology, Boston, MA, USA) [31]. Two shRNA target sequences specific for EpCAM (sh1, sh2) and a scrambled control sequence were cloned into the pSicoR-puromycin vector as previously described [32]. shRNA constructs were transfected into HEK293T cells with VSVG and $\Delta 8.9$, and viral supernatants were collected at 48 and 72 hours to transduce cells.

\section{Plasmids and transfection}

EpCAM cDNA was cloned from the MCF-7 breast cancer cell line. The c-Jun expression construct was obtained from Dr. R. Lee at the University of Virginia (Charlottesville, VA, USA). HA-MEKK1 was obtained from Dr. J. Avruch at Massachusetts General Hospital (Cambridge, MA, USA). Constructs from the JNK signal transduction pathway including pcDNA3 FLAG-MKK7/ JNK1, pcDNA3 FLAG-MKK7, and pcDNA3 FLAGJNK1 were obtained from Addgene (Cambridge, MA, USA). Cells were transfected with FuGENE-HD (Roche, Indianapolis, IN, USA) or Lipofectamine LTX (Invitrogen, Carlsbad, CA, USA) as recommended by the manufacturer.

\section{Invasion assay}

For invasion assays, stably transduced cells $\left(4 \times 10^{4}\right)$ were added to matrigel transwell invasion chambers or control transwell chambers (BD Biosciences San Jose CA, USA) and incubated for 24 to 72 hours with chemoattractant media (MEGM Clonetics, Walkersville, MD, USA) supplemented with growth factors. Cells invading through the matrigel or control membranes were fixed using $70 \%$ ethanol, stained with $0.1 \%$ crystal violet and photographed in four fields to cover the entire area. Cells were counted from all fields by a scientist blinded to the experimental conditions.

\section{Tumor challenge}

Six- to eight-week-old female nude mice were used for tumor challenge experiments. All mice were housed in pathogen-free facilities accredited by the Association for Assessment and Accreditation of Laboratory Animal Care. Animal use protocols were approved by the Washington University Animal Studies Committee. MDA-231 breast cancer cells stably transduced with scrambled control shRNA construct (SCR) and sh2 lentiviral shRNA constructs were harvested at 70 to $90 \%$ confluence. A $1 \times 10^{7}$ sample of MDA-231 cells were resuspended in matrigel and injected into nude mice in a total volume of $200 \mu \mathrm{L}$ ( $n=10$ per group). The right flank was injected with MDA-231(SCR) cells and the left flank was injected with MDA-231(sh2) cells. Tumor size was measured by electronic calipers every three to five days until 45 days. At the time of sacrifice, tumors were stored in formalin and submitted to an institutional core facility for H\&E staining and evaluation. 


\section{Cell cycle analysis and MTT proliferation assay}

Stably transduced MDA-231 cells were plated in culture media at $0.5 \times 10^{6}$ cells $/ \mathrm{mL}$. After 24 hours, the culture media was changed, and cells were pulse-labeled with BrdU (final concentration, $1 \mu \mathrm{M}$ ) for 30 minutes. Cells were washed with $1 \times$ PBS and fixed with $70 \%$ cold ethanol. For staining, cells were washed $1 \times$ with PBS with $0.1 \%$ BSA, and $0.1 \%$ Tween 20 and digested with DNAse for 30 minutes. The cells were washed again and then incubated with BrdU primary antibody (Becton Dickinson Immunocytometry Systems, San Jose, CA, USA) and fluorescein isothiocyanate (FITC) GAM secondary antibody (Becton Dickinson, San Jose, CA, USA). Cell cycle analyses were performed using the ModFit LT software (Topsham, ME, USA). For MTT assays, 5,000 cells were plated in 96-well plates in triplicate. After culture for the indicated time, MTT assays were preformed using a Vibrant MTT cell proliferation assay kit (Invitrogen, Carlsbad CA, USA). The optical density at 570 nm was measured using a microplate reader.

\section{Flow cytometry}

EpCAM expression levels were measured by flow cytometry using phycoerythrin-labeled EpCAM antibody with a FACScan flow cytometer (BD Biosciences, San Jose, CA, USA; \#347211). EpCAM expression was quantified as mean fluorescence intensity (MFI).

\section{Luciferase reporter assay}

The luciferase reporter constructs pTA-Luc (empty vector control), pTA-AP-1-Luc (AP-1 reporter), and pRLTK-Luc (transfection control) were obtained form Clontech Laboratories (Mountain View, CA, USA). A 400 ng sample of pTA-Luc or pTA-AP-1-Luc and $20 \mathrm{ng}$ of pRL-TK-Luc were transiently transfected into cell lines, plated in triplicate using either Lipofectamine-LTX (Invitrogen Carlsbad CA, USA) or FuGENE-HD (Roche, Indianapolis, IN, USA). The next day, cells were incubated with serum-free media. After 24 hours, cells were stimulated with phorbol-myristate acetate (PMA), and/ or epidermal growth factor (EGF) for an additional 16 hours as indicated. Reporter activity was determined using the Dual-Luciferase kit (Promega, Madison, WI, USA). Reporter activity was measured as luminescence using a luminometer and quantified as relative light units (RLU).

\section{cDNA synthesis and real-time RT-PCR analysis}

RNA was purified from cell lines using RNAeasy (Qiagen, Valencia, CA, USA). Three micrograms of RNA was reverse transcribed using a copy DNA (cDNA) synthesis kit (Ambion, Austin, TX, USA). Quantitative mRNA expression was measured using SYBR green chemistry and an ABI Prism 7700 Sequence Detector
(Applied Biosystems, Foster City CA, USA). Primer sequences of genes are available upon request. Each reaction was performed in triplicate, and the data is representative of two independent RNA preparations.

\section{Multiplex phosphoprotein assay}

For the multiplex phosphoprotein assay, CA1a cells were starved for 12 hours in serum-free media and treated with and without $100 \mathrm{ng} / \mathrm{mL}$ EGF for 15 minutes. Protein lysates were prepared using a cell lysis kit (BioRad, Hercules, CA, USA). Protein concentration was measured using a BCA protein assay (Pierce, Rockford, IL, USA). The presence of phosphorylated Akt (Ser473), c-Jun (Ser63/73), and JNK (Thr183/Tyr185) were detected by multiplex phosphoprotein assay (Bio-Rad, Hercules, CA, USA) according to the manufacturer's protocol. Data are expressed as mean \pm standard error of the mean of triplicate values from separate experiments.

\section{Immunoblots}

For phosphoprotein immunoblots, cells were cultured overnight in serum-free media and stimulated with 25 $\mu \mathrm{g} / \mathrm{mL}$ anisomycin for 30 minutes, washed with ice-cold PBS and lysed in cell lysis buffer (Cell Signaling Technology, Danvers, MA, USA). The protein concentration was measured using the BCA protein assay (Pierce, Rockford, IL, USA). A 30 to $50 \mu \mathrm{g}$ sample of protein was subjected to SDS-PAGE (NuPAGE, Invitrogen, Carlsbad, CA, USA), and transferred by electrophoresis to a polyvinylidene fluoride (PVDF) membrane. Antibodies were obtained from Santa Cruz Biotechnology (Santa Cruz, CA, USA) (EpCAM, \#sc-25308; JNK, \#sc571, \#sc-474; c-Jun, \#sc-1694; HA, \#sc-7392; actin, \#sc1615), Sigma-Aldrich (Saint Louis, MO, USA) (FLAG, \#F3165), and Cell Signaling Technology (Danvers, MA, USA) (phospho-JNK, \#9255; phospho-c-Jun ser73, \#9164; SOD1, \#2770). Signal detection was performed using the ECL chemiluminescent immunodetection system (Applied Biosystems, Foster City, CA, USA). Immunoblot band density was analyzed using a Bio-Rad GS800 calibrated densitometer (Bio-Rad, Hercules, CA, USA). For JNK immunoprecipitation, confluent cells were serum starved overnight and immunoprecipitation was carried out as described [33]. To quantify band density, immunoblots were developed on film and then scanned and analyzed using ImageJ software (National Institutes of Health, USA; http://rsbweb.nih.gov/ij/). Plots of each lane were generated, and the area under the peak corresponding to control samples (SCR) was determined, and arbitrarily set at 1.0. The area under the peak corresponding to experimental samples (sh2) was then determined, and expressed relative to the corresponding control. 


\section{Statistical analysis}

The data are given as the mean values \pm standard deviation. Statistical significance was evaluated by the Student's $t$ test. $P$ values less than 0.05 were considered to be statistically significant. Significant results are indicated in the appropriate figures with an asterisk.

\section{Results}

\section{EpCAM expression is associated with breast cancer} invasion in vitro and in vivo

We have previously reported that specific ablation of EpCAM decreases breast cancer invasion in vitro $[4,7,32]$. Many of these studies were performed with siRNA, and the short duration of RNA interference associated with these reagents precluded in vivo and/or mechanistic experiments. To confirm the impact of EpCAM on breast cancer invasion in vivo, and to establish a functional rescue/gain-of-function model system for mechanistic studies, we generated stable breast cancer cell lines with specific ablation of EpCAM expression using EpCAM-specific lentiviral shRNA constructs. Stable transduction of breast cancer cell lines with EpCAM-specific lentiviral shRNA constructs results in more than $90 \%$ reduction in EpCAM protein expression as measured by immunoblot (Figure 1a). Stable specific ablation of EpCAM in both MDA-231 and CA1a breast cancer cell lines is also associated with a more than $80 \%$ decrease in invasion in matrigel transwell invasion assays (Figure 1b).

To control for off-target effects of RNA interference, and establish a functional rescue model system for
A.

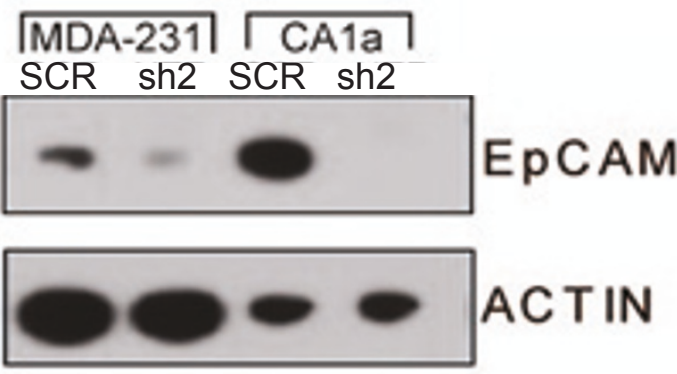

C.

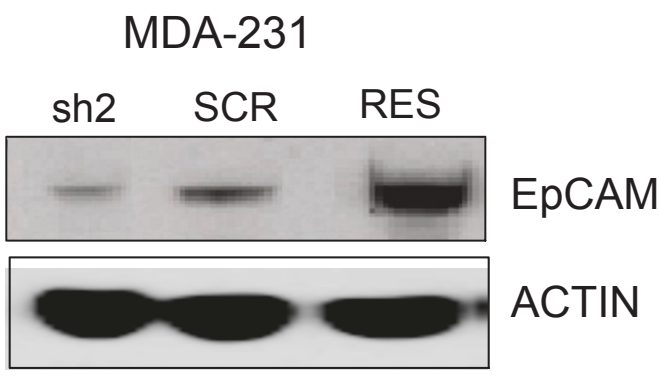

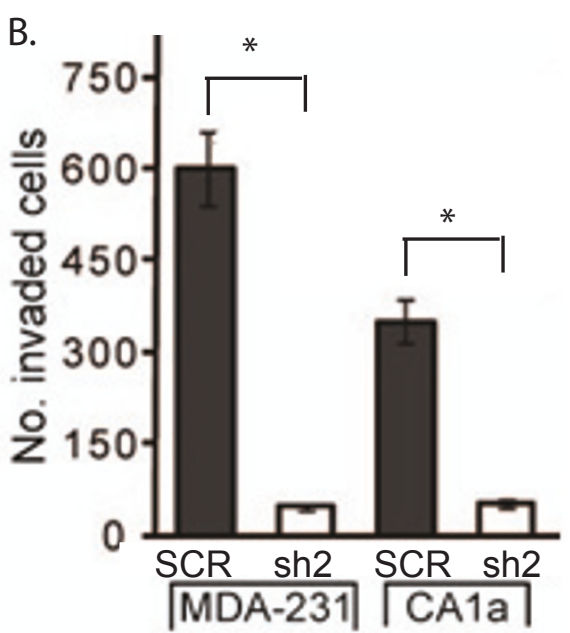

D.

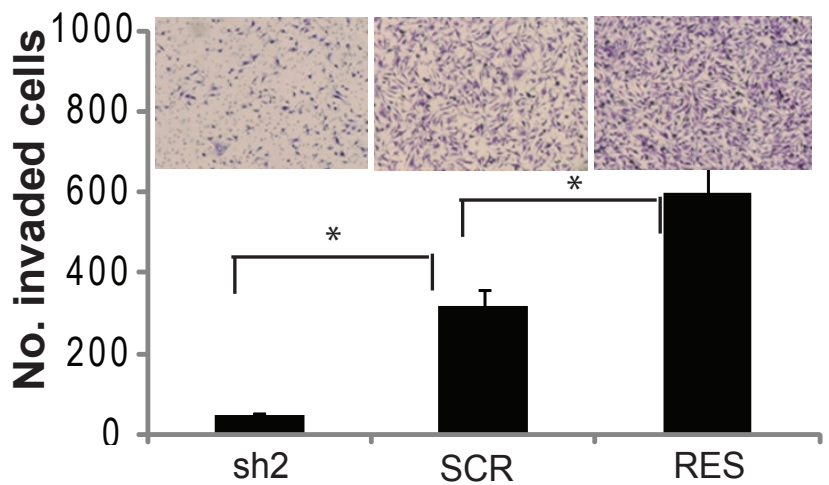

Figure 1 EpCAM expression is associated with breast cancer invasion in vitro. Epithelial cell adhesion molecule (EpCAM) expression was specifically ablated in the breast cancer cell lines MDA-231 and CA1a by RNA interference and rescued, as indicated, by transient transfection with an EpCAM cDNA resistant to RNA interference. (a) Specific ablation of EpCAM by shRNA results in a significant decrease in EpCAM protein expression as measured by immunoblot. For each cell line, sh2 designates transduction with a lentiviral vector targeting the $3^{\prime}$ untranslated region of EPCAM, while scrambled control shRNA construct (SCR) designates transduction with a lentiviral vector expressing a scrambled control shRNA sequence. (b) Invasion was measured using a matrigel transwell invasion assay. After 24 to 72 hours, cells invading through the matrigel membranes were fixed using 70\% ethanol, stained with $0.1 \%$ crystal violet and photographed in four fields to cover the entire area. Error bars represent the standard error of three experiments performed in triplicate. (c and $\mathbf{d}$ ) Rescue of EpCAM expression in MDA-231 cells by cDNA expression (RES), rescues protein expression (immunoblot) and invasion (matrigel transwell invasion assay). 
A.

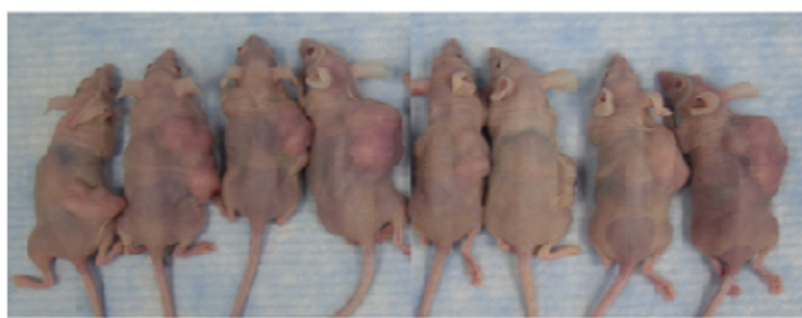

Right-SCR Left- sh2
B.

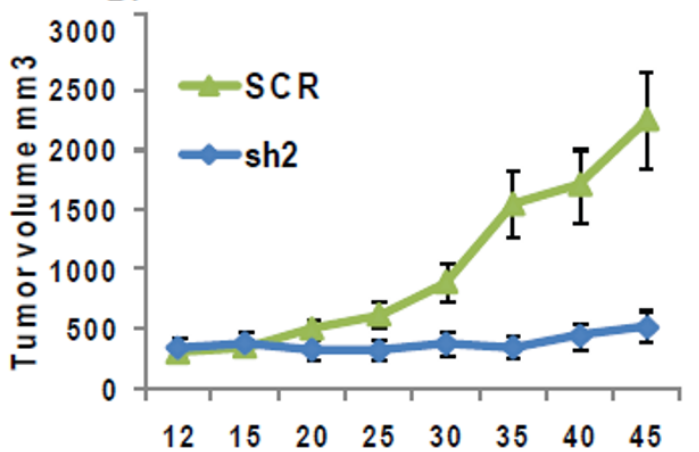

c.
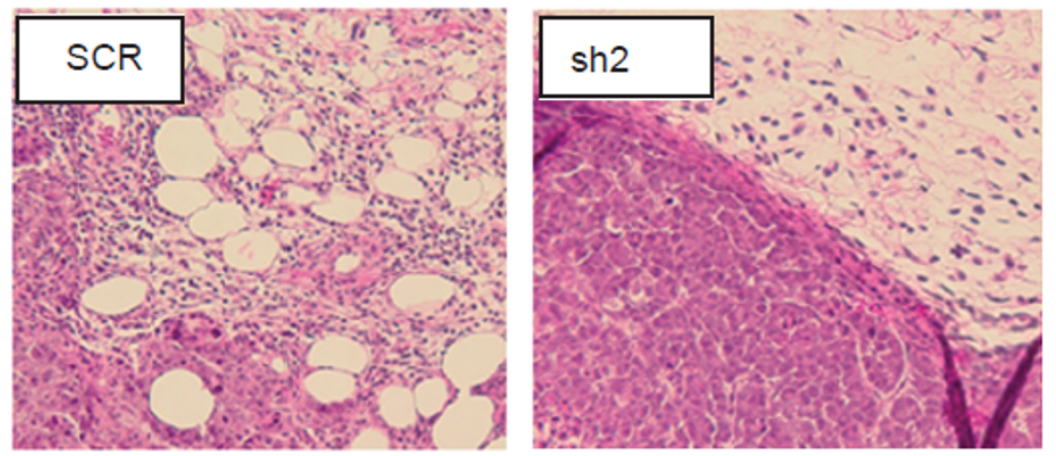

D. SCR

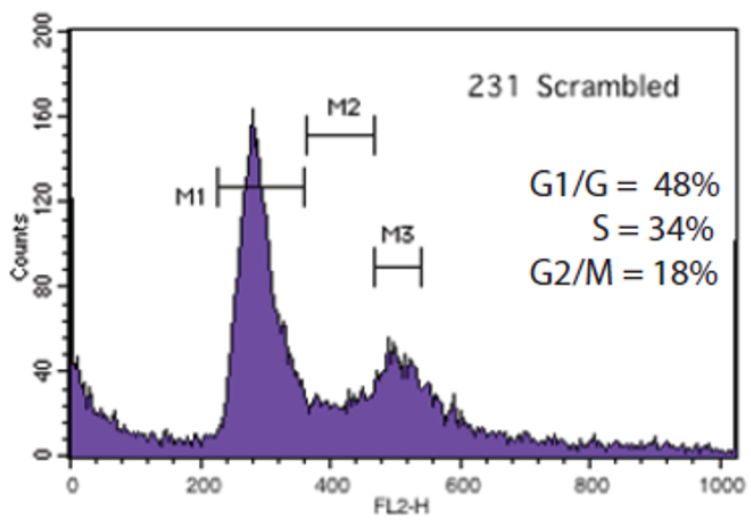

$\operatorname{sh} 2$

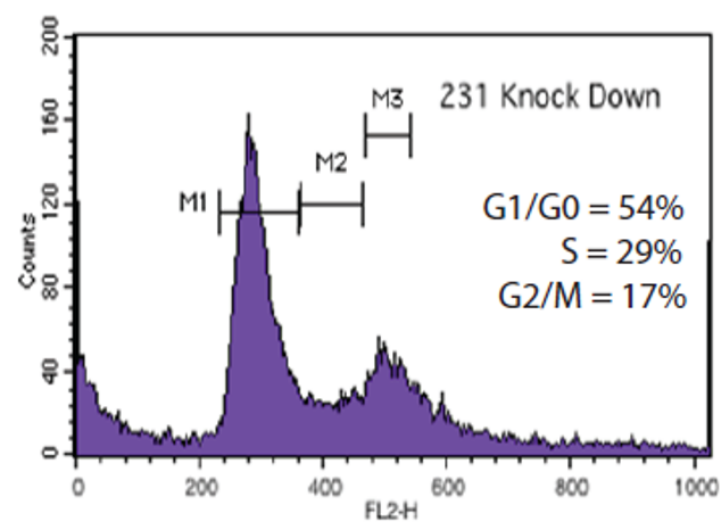

Figure 2 EpCAM expression is associated with breast cancer invasion in vivo in a breast cancer xenograft model. (a and b) MDA-231 breast cancer cells were stably transduced with experimental (sh2) or control (scrambled control shRNA construct (SCR)) shRNA lentiviral constructs. One $\times 10^{7} \mathrm{MDA}-231$ cells were resuspended in matrigel (1:1 ratio by volume) and injected into nude mice ( $n=10$ per group). The right flank was injected with MDA-231(SCR) cells and the left flank was injected with MDA-231(sh2) cells. Tumor size was measured by electronic calipers every thre to five days. Photos at the time of sacrifice demonstrate tumor growth in the right flank but not in the left flank. (c) H\&E evaluation of the tumor site demonstrates that MDA-231(SCR) cells were invading into the surrounding fat, while MDA-231(sh2) tumors were not invasive. (d) Specific ablation of EpCAM did not impact cell cycle/proliferation of MDA-231 cells when analyzed prior to tumor challenge. The percentage of cells in each stage of the cell cycle is indicated. 
mechanistic studies, we specifically ablated EpCAM expression using specific shRNA constructs and then rescued EpCAM expression using EpCAM cDNA constructs resistant to RNA interference as we previously described [32] (Figures 1c and 1d). In these experiments, EpCAM rescue in MDA-231 and CA1a breast cancer cells results in EpCAM overexpression as measured by immunoblot, and increased invasion, establishing a gainof-function model system.

We used the lentiviral vectors validated above to determine the impact of EpCAM expression on breast cancer invasion in vivo, taking advantage of a commonly used breast cancer xenograft model [34-36]. Specific ablation of EpCAM expression in MDA-231 breast cancer cells significantly decreases tumor growth following tumor challenge in nude mice (Figures $2 \mathrm{a}$ and $2 \mathrm{~b}$ ). Histological examination of the tumors confirms that specific ablation of EpCAM abrogates the ability of tumors to invade into surrounding tissues (Figure 2c). Of note, we and others have observed that depending on the experimental conditions, EpCAM expression can impact proliferation [3-5]. Significant changes in proliferation could impact interpretation of the in vivo tumor challenge studies. However, only minimal changes in cell cycle (Figure 2d) and proliferation (data not shown) were observed following specific ablation of EpCAM expression in MDA-231 breast cancer cells under these experimental conditions.

Recently, Gires et al. demonstrated that EpCAM is cleaved by regulated intramembrane proteolysis, resulting in release of the extracellular domain as a soluble ligand, and translocation of the intracellular domain (EpICD) into the nucleus [5,37]. In these studies, the extracellular domain of EpCAM is also able to modulate EpCAM signaling. We used recombinant soluble extracellular EpCAM (rEpCAM) to rescue EpCAM signaling following specific ablation of EpCAM. Addition of rEpCAM to MDA-231 breast cancer cells is able to rescue invasion following specific ablation of EpCAM (Figure 3). Taken together, these findings confirm the hypothesis that EpCAM expression modulates breast cancer invasion, with increased EpCAM expression and/ or signaling associated with increased breast cancer invasion.

\section{EpCAM expression is associated with increased AP-1 transcription factor activity in breast cancer cells}

To address the potential mechanism(s) underlying the impact of EpCAM on breast cancer invasion, we used a luciferase reporter pathway profiling system to measure the activity of specific transcription factors following specific ablation of EpCAM expression. In this system, individual vectors in the profiling system contain the transcriptional response elements of the transcription factors of interest upstream of a luciferase reporter. These reporters are then individually transfected with a control TK-Renilla construct into cells stably transduced with control or EpCAM-specific shRNA vectors. In these studies, we focused on the AP-1 transcription factor, because AP-1 has been shown to be a critical regulator of a complex program of gene expression contributing to the invasive phenotype [38]. Using this strategy, we found that specific ablation of EpCAM is associated with a two-fold or greater reduction in AP-1 transcription factor activity in MDA-231 breast cancer cells at baseline (Figure 4a), or in the presence of EGF, or PMA, which are known to induce AP-1 activity [39] (Figure 4b). Rescue of EpCAM expression using EpCAM cDNA resistant to RNA interference, or rescue of EpCAM signaling using recombinant soluble EpCAM resulted in a dose-dependent rescue of AP-1 transcription factor activity, confirming the specificity and significance of these observations (Figures $4 \mathrm{c}$ and $4 \mathrm{~d}$ ).

\section{EpCAM expression is associated with increased phosphorylation of the AP-1 protein subunit c-Jun}

AP-1 can be activated through at least two possible regulatory mechanisms $[40,41]$. First, transcription of c-Jun and other AP-1 protein subunits can be rapidly induced, resulting in increased total protein levels. Second, AP-1 protein subunits can be phosphorylated by mitogen-activated protein kinases (MAPKs), including JNK. In preliminary studies using a multiplex phosphoprotein assay, specific ablation of EpCAM decreased c-Jun and JNK1 phosphorylation in breast cancer cells (Figure 5a). To confirm and extend these findings, we specifically ablated EpCAM in breast cancer cells (CA1a, and MCF-

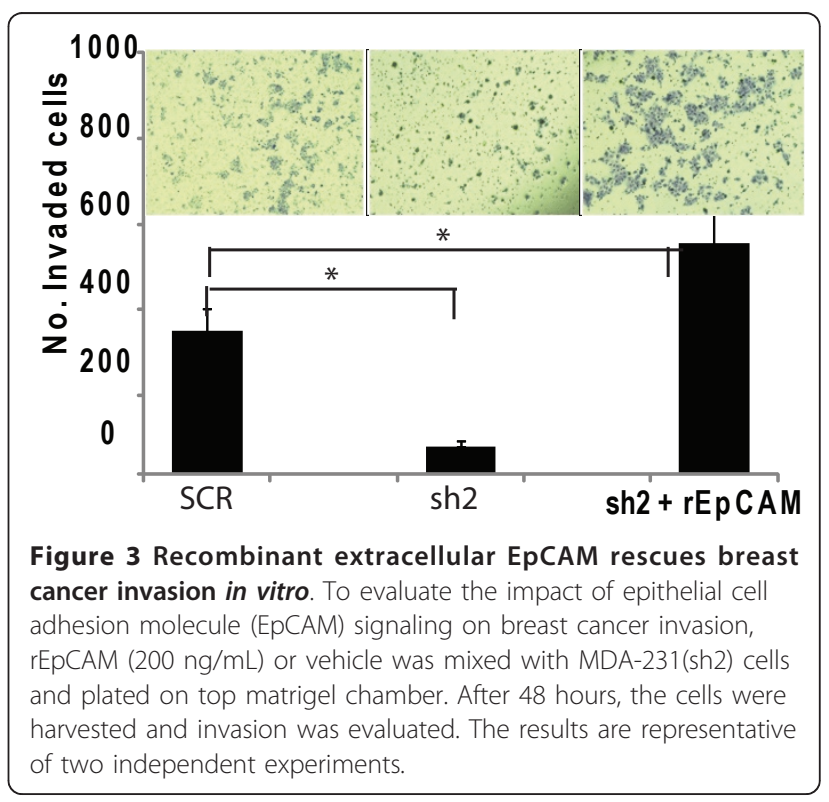


A.

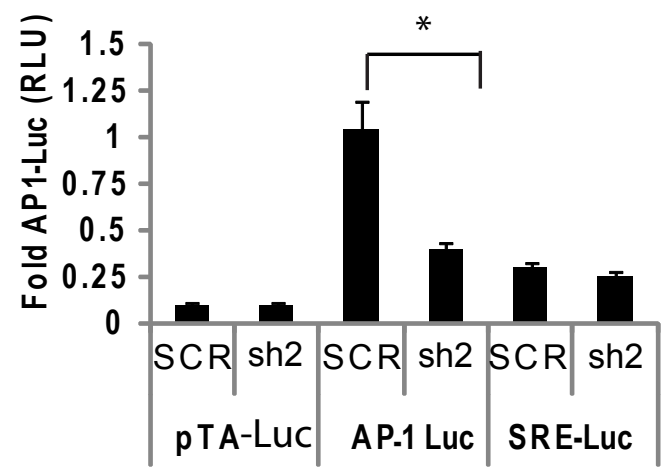

C.

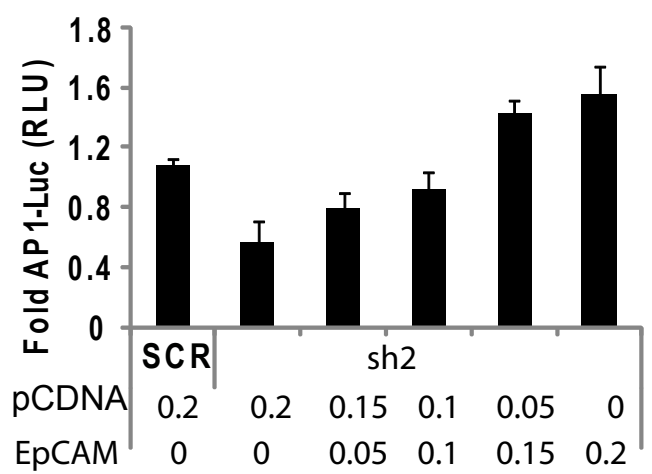

B.

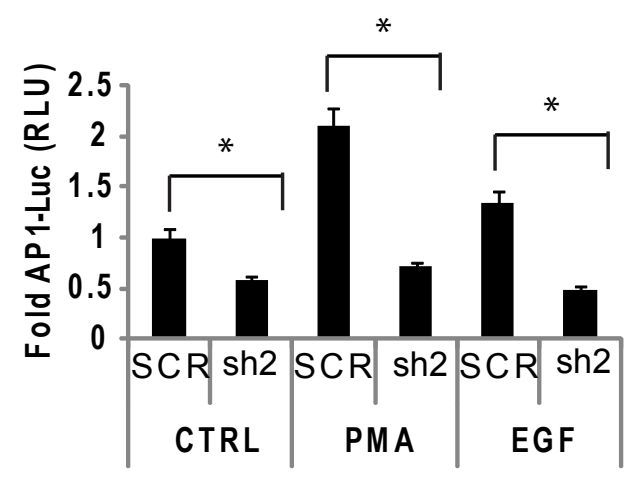

D.

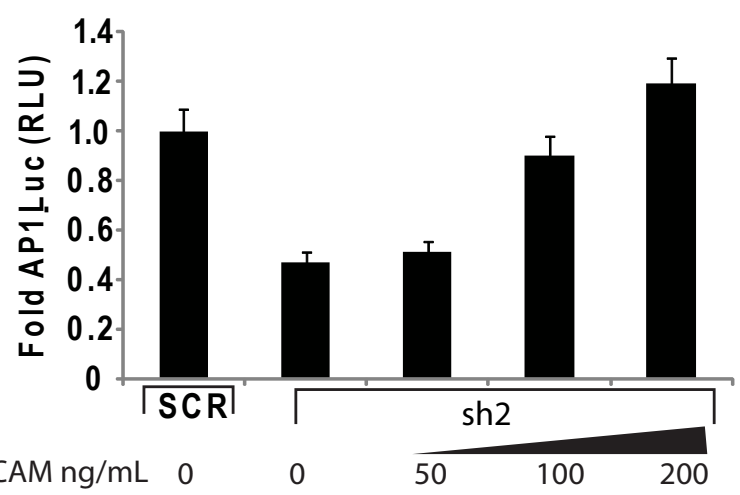

Figure 4 EpCAM expression is associated with AP-1 transcription factor activity. Epithelial cell adhesion molecule (EpCAM) expression was specifically ablated in breast cancer cells using lentiviral constructs, and then rescued by cDNA or recombinant EpCAM. (a) A luciferase reporter pathway profiling system was used to measure the impact of EpCAM expression on the activity of specific transcription factors. Results from the PTA (control), activator protein 1 (AP-1) and SRE reporters are shown. AP-1 transcription factor activity was significantly decreased following specific ablation of EpCAM in MDA-231(sh2) cells. Other transcription factors were evaluated but specific ablation of EpCAM had minimal effect (data not shown). Stably transduced MDA-231 cells (scrambled control shRNA construct (SCR) and sh2) were plated for 12 hours, then transiently transfected with the reporters indicated. Luciferase activity was measured 48 hours later. (b) Following this same protocol, the AP-1 reporter was transfected into stably transduced MDA-231 cells (SCR and sh2). Twelve hours after transfection, AP-1 transcription factor activity was induced with phorbol-myristate acetate (PMA; 50 ng/mL) or epidermal growth factor (EGF; 10 ng/mL). 12 hours after induction, reporter activity was measured. (c) AP-1 transcription factor activity is rescued in a dose-dependent fashion by transfection with increasing concentrations of EpCAM cDNA. MDA-231(sh2) cells were transiently transfected with 200 ng of pcDNA3, or 50 to 200 ng of pCDNA-EpCAM as indicated. The total amount of DNA was constant at 200 ng, as indicated. (d) AP-1 transcription factor activity is rescued in a dose-dependent fashion by recombinant EpCAM. Twelve hours after transient transfection with the AP-1 reporter, the indicated doses of rEpCAM were added to MDA-231 (sh2) cells. Fourteen hours after addition of rEpCAM, AP-1 reporter activity was measured. The results are representative of at least two independent experiments.

7), and then measured the levels of total and phosphorylated c-Jun by protein immunoblot and densitometry. In CA1a and MCF-7 breast cancer cells, specific ablation of EpCAM decreased phosphorylated c-Jun, but had no significant impact on total c-Jun (Figure 5b). A similar decrease in phosphorylated but not total ATF2 was also observed (data not shown). We also evaluated total and phosphorylated c-Jun following rescue of EpCAM signaling by $\mathrm{rEpCAM}$. Increasing doses of rEpCAM rescued phosphorylated c-Jun following specific ablation of EpCAM in CA1a cells (Figure 5c). Taken together, these studies suggest that phosphorylation of existing AP-1 protein subunits contributes to modulation of AP-1 transcription factor activity following specific ablation or rescue of EpCAM expression and/or signaling.

\section{The JNK signal transduction pathway contributes to EpCAM-dependent AP-1 transcription factor activity} AP-1 is a major target of the JNK signal transduction pathway, and activated JNK can bind and phosphorylate c-Jun. To directly evaluate the impact of EpCAM expression on JNK phosphorylation, we manipulated EpCAM expression and then measured JNK phosphorylation by immunoblot and densitometry. Specific 
A.

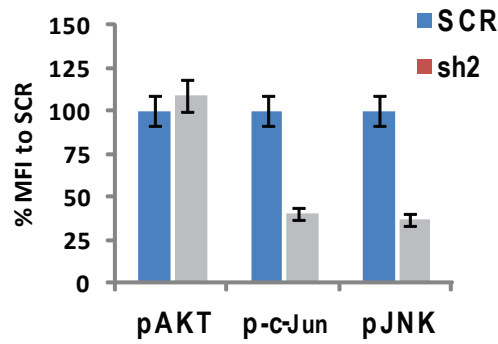

B.

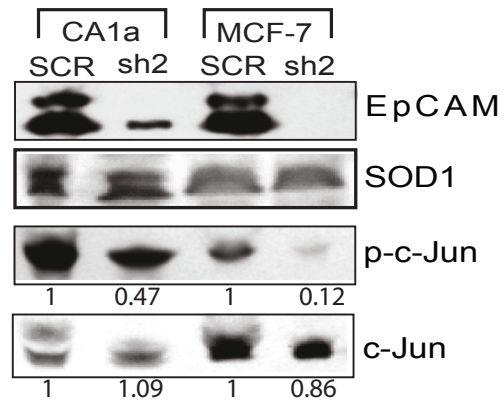

C.

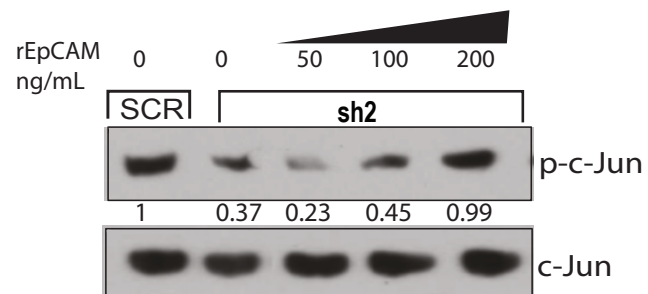

Figure 5 EpCAM expression is associated with phosphorylation of the AP-1 transcription factor protein subunit c-Jun. (a) Specific ablation of epithelial cell adhesion molecule (EPCAM) is associated with decreased c-Jun phosphorylation as measured by multiplex phosphoprotein assay. Stably transduced CA1a cells (scrambled control shRNA construct (SCR) and sh2) were induced with epidermal growth factor (EGF), and phosphorylation was measured using a multiplex phosphoprotein assay. Relative levels of phosphorylated ( $p$ )-AKT, $p$-c-Jun, and p-JNK were measured by flow cytometry and expressed as mean fluorescence intensity (MFI). Error bars represent standard error. (b) Specific ablation of EpCAM is associated with decreased c-Jun phosphorylation as measured by phosphoprotein immunoblot. Stably transduced CA1a and MCF-7 cells (SCR and sh2) were serum-starved for six hours. The cells were treated with $20 \mu \mathrm{M}$ anisomycin for 15 minutes and $30 \mu \mathrm{g}$ of the cell lysate was analyzed with antibodies recognizing EpCAM, superoxide dismutase (SOD, loading control), p-c-Jun-63, or total c-Jun. Relative band density was quantified using ImageJ software, with the results indicated below the relevant band. (c) Recombinant EpCAM is capable of rescuing c-Jun phosphorylation in CA1a(sh2) cells. CA1a (sh2) cells were serum-starved for six hours and then treated with rEpCAM at concentrations of 50, 100, and $200 \mathrm{ng} / \mathrm{mL}$ for two hours. The cells were then stimulated with anisomycin for 10 minutes. A $30 \mu \mathrm{g}$ sample of each cell lysate was analyzed for p-c-Jun and total c-Jun. Relative band density was quantified using ImageJ software, with the results indicated. The results are representative of two independent experiments. ablation of EpCAM results in a significant decrease in JNK phosphorylation in CA1a and MCF-7 breast cancer cells (Figure 6a). To evaluate the contribution of the JNK signal transduction pathway in more detail, we used pharmacologic inhibitors of the JNK signal transduction pathway. Specific ablation of EpCAM is associated with a decrease in AP-1 transcription factor activity, and this decrease can be rescued in a dosedependent fashion with rEpCAM (Figure 6a). Addition of SP600125, an anthrapyrazolone inhibitor of JNK [42], abrogates the increase in AP-1 transcription factor activity induced by $\mathrm{rEpCAM}$, suggesting a role for the JNK signal transduction pathway. To confirm and extend these findings we used constitutively active genetic constructs corresponding to MAP kinases in the JNK signal transduction pathway (MEKK1-MKK7-JNK). Constitutively active JNK MAPK constructs rescued c-Jun phosphorylation (Figure 6c) and AP-1 transcription factor activity (Figure 6d) following specific ablation of EpCAM in MCF-7 breast cancer cells. Taken together these studies provide evidence that EpCAM expression can modulate the JNK signal transduction pathway, and AP-1 transcription factor activity.

\section{AP-1 contributes to EpCAM-mediated breast cancer invasion}

AP-1 is considered to be a central transcription factor in the regulation of cancer invasion [38]. To directly assess the functional contribution of AP-1 in EpCAMmediated breast cancer invasion, we specifically ablated EpCAM expression in CA1a breast cancer cells, and then rescued AP-1 transcription factor activity using a constitutively active c-Jun genetic construct [43]. c-Jun expression is able to efficiently rescue AP-1 transcription factor activity (data not shown) and invasion (Figure 7a), suggesting that the AP-1 protein subunit c-Jun is a key downstream mediator of EpCAM biology, contributing to EpCAM-dependent breast cancer invasion.

\section{EpCAM expression modulates the expression of AP-1 target genes known to be involved in cancer invasion} To confirm the impact of EpCAM on AP-1 target gene expression, we specifically ablated EpCAM and then analyzed more than $120 \mathrm{AP}-1$ target genes known to be involved in cancer invasion using quantitative RT-PCR [38]. We identified more than 30 genes that were impacted at the mRNA level by specific ablation of EpCAM. To confirm these results, EpCAM expression was specifically ablated in CA1a breast cancer cells, and then rescued with increasing doses of EpCAM cDNA. Increasing EpCAM expression is associated with a dosedependent increase in the expression of the AP-1 target genes CTSL1, Autotaxin and ARP2/3 (Figure 8). These studies provide additional support for the hypothesis 
A.

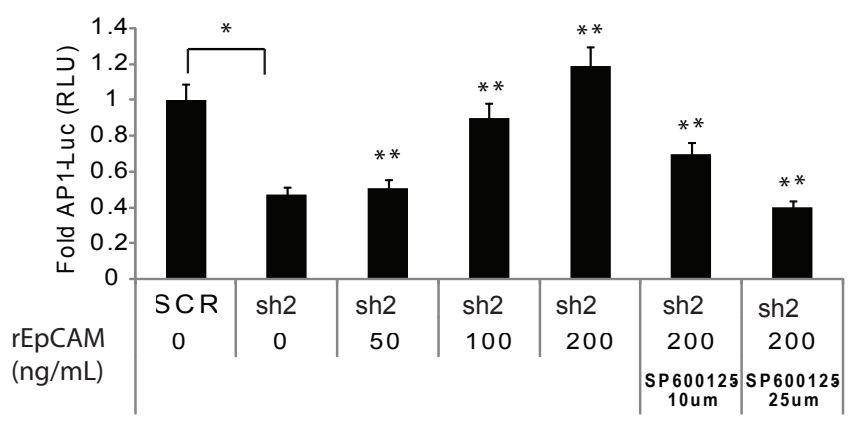

B. IP: JNK IB JNK.

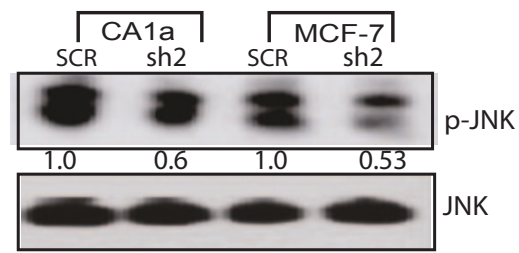

C.

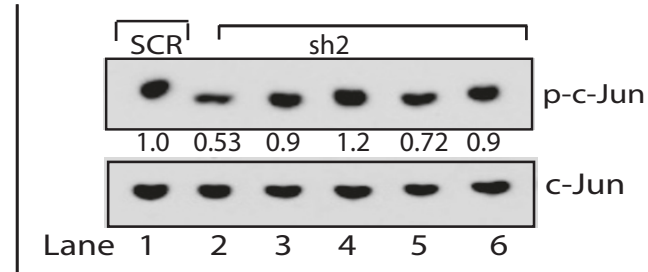

D.

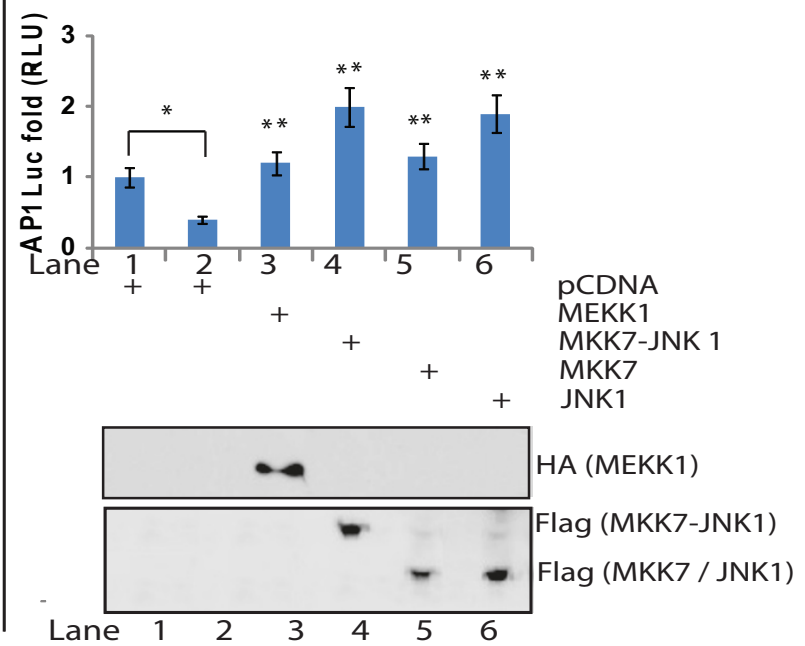

Figure 6 The MEKK1/MKK7/JNK signal transduction pathway contributes to EpCAM-dependent AP-1 transcription factor activity. (a) Pharmacologic inhibition of c-Jun N-terminal kinase (JNK) abrogates recombinant soluble extracellular epithelial cell adhesion molecule (rEpCAM)-mediated rescue of activator protein 1 (AP-1) transcription factor activity. Stably transduced CA1a (scrambled control shRNA construct (SCR) and sh2) cells were transiently transfected with AP-1 and control luciferase reporters. Six hours after transfection, cells were treated with SP600125 (JNK inhibitor) at $10 \mu \mathrm{M}$ and $20 \mu \mathrm{M}$ for one hour followed by addition of rEpCAM. Luciferase reporter activity was measured 16 hours later. (b) To directly evaluate JNK phosphorylation, SCR and sh2 CA1a and MCF-7 cells were serum starved for 12 hours, stimulated with $20 \mu \mathrm{M}$ anisomycin for 10 minutes, and then immunoprecipitated overnight with a JNK antibody. JNK phosphorylation was evaluated by immunoblot using antibodies specific for p-JNK1/2 and total JNK1. Relative band density was quantified using ImageJ software, with the results indicated. (c) Constitutively active genetic constructs corresponding to mitogen activated protein (MAP) kinases in the JNK signal transduction pathway rescued c-Jun phosphorylation and AP-1 transcription factor activity following specific ablation of EpCAM in MCF-7 breast cancer cells. Stably transduced SCR or sh2 MCF-7 cells were transiently transfected with plasmids encoding HA-MEKK1, FLAG-MKK7-JNK1, FLAG-MKK7, and FLAGJNK1. After 16 hours, cells were analyzed by immunoblot for c-Jun phosphorylation. Relative band density was quantified using ImageJ software, with the results indicated. (d) Duplicate samples were transiently transfected with control and AP-1 luciferase reporters, and AP-1 transcription factor activity was measured. (e) Expression of HA-MEKK1, FLAG-MKK7-JNK1, FLAG-MKK7, and FLAG-JNK1 was confirmed by immunoblot with antibodies specific for the indicated protein tag (HA or FLAG). The results are representative of two independent experiments. For AP-1 reporter assay in Figure a and $\mathrm{d}, P<0.05$ were considered to be statistically significant when compared between SCR, sh2 $\left(^{*}\right)$ and sh2, sh2 treated/ transfected cells $\left({ }^{*}\right)$. 
A.

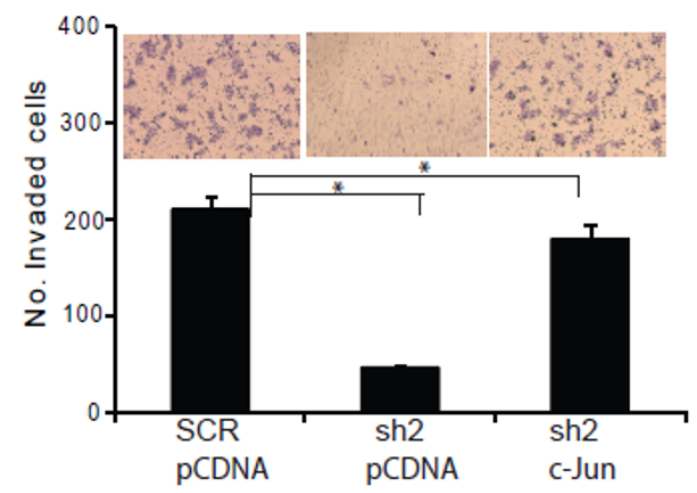

B.

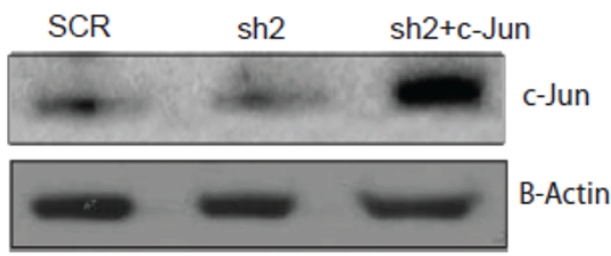

Figure 7 Forced overexpression of c-Jun rescues breast cancer invasion in vitro following specific ablation of EpCAM. (a) Stably transduced CA1a cells (sh2), were transiently transfected with pcDNA or c-Jun constructs. After 24 hours, invasion was assessed using a matrigel transwell invasion assay. (b) Transfected cells were analyzed for c-Jun mRNA expression by RT-PCR. Actin mRNA expression served as a control for RT-PCR conditions. The results are representative of two independent experiments. EpCAM, epithelial cell adhesion molecule.

that EpCAM expression modulates AP-1 transcription factor activity.

\section{Discussion}

EpCAM is a cell-surface glycoprotein that is overexpressed on the majority of epithelial cancers, including breast cancer. However, the functional role of EpCAM in cancer invasion remains controversial. In this study we focused on the role of EPCAM in breast cancer invasion. We confirm that EpCAM expression is associated with increased breast cancer invasion in vitro and in vivo, and demonstrate for the first time that EpCAM expression is capable of modulating the JNK/AP-1 signal transduction pathway. In functional rescue experiments, we demonstrate that forced overexpression of c-Jun is capable of rescuing breast cancer invasion following specific ablation of EpCAM, confirming that AP-1 is a key downstream mediator of EpCAM-dependent breast cancer invasion.

Recently, Munz et al. demonstrated that de novo induction of EpCAM expression is associated with the

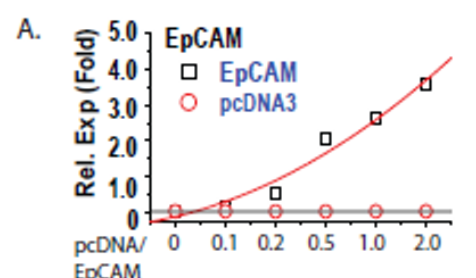

B.

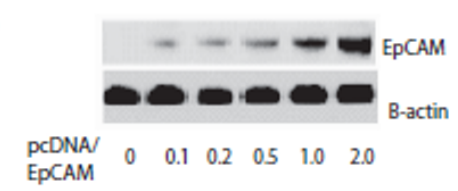

C.

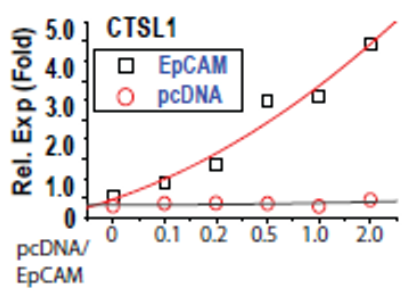

D.
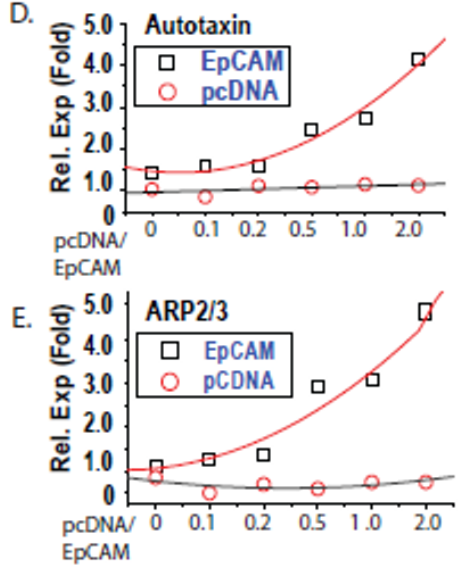

Figure 8 EpCAM expression modulates expression of AP-1 target genes known to be involved in cancer invasion. MDA231 cells were transiently transfected with control and epithelial cell adhesion molecule (EPCAM) CDNA expression constructs and allowed to stabilize for 24 hours. The cells were then serum-starved for 16 hours, and then stimulated with 10\% FBS DMEM growth media for two hours. RNA was extracted using RNeasy (Qiagen). Two micrograms of RNA was reverse transcribed and $40 \mathrm{ng}$ equivalent CDNA was used for each assay in triplicate. Activator protein 1 (AP-1) target genes CTSL1, Autotaxin, and ARP2/3 were analyzed using real-time PCR. Relative expression was quantified using the [delta] [delta] Ct method with 185 rRNA as a control. The results are representative of at least two independent experiments.

rapid upregulation of c-myc expression in epithelial cells, strongly suggesting that EpCAM can function as a signaling molecule [3]. In their studies the most dramatic phenotype of forced EpCAM expression was on cell cycle and proliferation, consistent with the known 
role of c-myc in regulating genes involved in control of the cell cycle. However, we and others have found that the most dramatic phenotype associated with manipulation of EpCAM expression in human cancers is altered invasion $[4,7,29,30]$. This suggests that other signaling pathways may also be modulated by EpCAM expression/signaling. Our finding that EpCAM differentially regulates the JNK/AP-1 signal transduction pathway is particularly relevant in this context. The AP-1 transcription factor is a heterodimeric protein composed of proteins belonging to the c-Jun, c-Fos, ATF, and JDP families [39]. AP-1 is activated in response to a variety of stimuli, including cytokines, growth factors, stress, and infection, and is considered to be a central transcription factor in the regulation of cell invasion [38]. Of particular note, AP-1 appears to be overexpressed in breast cancer [44,45], and is currently being evaluated as a target for molecular therapy in this disease [46-48]. Recent studies have focused specifically on the role of cJun in breast cancer. In primary breast cancers, activated c-Jun is present at the invasive front, and is associated with proliferation and angiogenesis [45]. In addition, recent studies confirm the role of c-Jun in breast cancer invasion in vitro [49], and in spontaneous tumors derived from genetically engineered mice [50]. Despite the central role of AP-1 as a regulator of invasion, our studies do not exclude the possibility that other signaling pathways are modulated by EpCAM, and may also contribute to the regulation of invasion in breast cancer. Recently, Gostner et al. demonstrated that specific ablation of EpCAM in MDA-231 cells may modulate the Wnt signaling pathway [51].

Maetzel et al. recently demonstrated that EpCAM is cleaved by regulated intramembrane proteolysis in a hypopharyngeal cancer cell line, resulting in the release of the extracellular portion of the molecule, $\mathrm{Ep}^{\mathrm{EX}}$, and the intracellular domain, $\mathrm{Ep}^{\mathrm{IC}}$ [5]. In their studies, $\mathrm{Ep}^{\mathrm{IC}}$ localizes to the nucleus, and interacts with FHL2 and $\beta$ catenin to promote Wnt signaling. Our studies confirm the importance of the extracellular domain of EpCAM in EPCAM signaling; addition of recombinant extracellular EpCAM rescues breast cancer invasion, AP-1 transcription factor activity, and c-Jun phosphorylation in a dose-dependent fashion following specific ablation of EpCAM. However, we have been unable to rescue invasion by forced overexpression of $\mathrm{Ep}^{\mathrm{IC}}$ (data not shown). FHL2 is known to be an inducible co-activator of AP-1 [52], and one hypothesis is EpCAM expression/signaling results in $\mathrm{Ep}^{\mathrm{IC}}$ translocation to the nucleus and subsequent interaction with FHL2 and AP-1 proteins to increase AP-1 signaling. Alternatively, EpCAM expression/signaling may modulate the JNK signal transduction pathway directly or indirectly, leading to c-jun phosphorylation and AP-1 activation. In support of this latter hypothesis, cell adhesion molecules such as ICAM-1 are known to modulate the JNK/AP-1 signaling pathway. Although our data does suggest that the JNK/ AP-1 signaling pathway is modulated, these hypotheses are not mutually exclusive, and additional studies are ongoing to determine how EpCAM signaling impacts AP-1 transcription factor activity.

Finally, EpCAM represents an attractive target for molecular therapy in epithelial carcinomas. EpCAM is overexpressed in the majority of epithelial carcinomas, and there are currently a number of different molecular therapies under development [1]. The results of the studies presented here have important implications for the design and application of these molecular therapies. The most important implication is that molecular therapies under investigation should be evaluated for their ability to influence EpCAM-mediated signaling pathways, as recent studies suggest that monoclonal antibodies targeting EpCAM may alter EpCAM signaling [53]. Molecular therapies capable of interfering with EpCAM-mediated signaling may find particular success in the treatment of breast cancer, where EpCAM expression is associated with increased invasion and poor prognosis.

\section{Conclusions}

EpCAM expression is associated with increased breast cancer invasion. In mechanistic studies, we demonstrate that EpCAM expression modulates the JNK signal transduction pathway, and AP-1 transcription factor activity in breast cancer cells. Functional studies confirm that AP-1 is a key downstream mediator of EpCAM biology, contributing to EpCAM-dependent breast cancer invasion. Further study of EpCAMmediated signaling pathways is necessary to facilitate the rational design and application of molecular therapies targeting EpCAM.

\section{Abbreviations \\ AP-1: activator protein 1; BSA: bovine serum albumin; CDNA: copy DNA; EGF: epidermal growth factor; EpCAM: epithelial cell adhesion molecule; Ep ${ }^{\mathrm{EX}}$ : EpCAM extracellular domain; Ep ${ }^{1 C}$ : EpCAM intracellular domain; H\&E: hematoxylin and eosin; JNK: c-Jun N-terminal kinase; MAPK: mitogen activated protein kinase; MFI: mean fluorescent intensity; PBS: phosphate- buffered saline; PMA: phorbol-myristate acetate; rEpCAM: recombinant soluble extracellular EpCAM; RLU: relative light unit; RT-PCR: reverse transcription polymerase chain reaction; SCR: scrambled control shRNA construct; shRNA: short hairpin RNA; sh2 = shRNA construct targeting the untranslated region of EpCAM mRNA.}

\section{Acknowledgements}

Support for this study was provided by Susan G. Komen for the Cure. The authors thank Jason Weber for helpful discussions.

\section{Author details}

${ }^{1}$ Department of Surgery, Washington University School of Medicine, Campus Box 8109, 660 South Euclid Avenue, St. Louis, Missouri, 63110, USA. ${ }^{2}$ The Alvin J. Siteman Cancer Center at Barnes-Jewish Hospital and Washington 
University School of Medicine, 660 South Euclid Avenue, St. Louis, Missouri, 63110, USA

\section{Authors' contributions}

NVS contributed to the conception of the study, performed the majority of the molecular biology studies, and wrote the first draft of the manuscript. JDM performed some of the phosphorylation studies and assisted in other molecular biology experiments. MWW participated in the rEpCAM signaling experiments and assisted in cloning many of the constructs used in the study. TPF performed the invasion assays, contributed to study conception, data interpretation and critical manuscript review. WEG contributed to study conception, data interpretation, troubleshooting, and edited the manuscript. All authors read and approved the final manuscript.

\section{Authors' information}

JDM is currently a post-doctoral fellow at the Duke Institute for Genome Sciences \& Policy (IGSP). MWW is currently a medical student at Saint Louis University.

\section{Competing interests}

The authors declare that they have no competing interests.

Received: 28 February 2011 Revised: 2 November 2011

Accepted: 1 December 2011 Published: 1 December 2011

\section{References}

1. Baeuerle PA, Gires O: EpCAM (CD326) finding its role in cancer. $\mathrm{Br} J$ Cancer 2007, 96:417-423.

2. Litvinov SV, Bakker HA, Gourevitch MM, Velders MP, Warnaar SO: Evidence for a role of the epithelial glycoprotein 40 (Ep-CAM) in epithelial cell-cel adhesion. Cell Adhes Commun 1994, 2:417-428.

3. Munz M, Kieu C, Mack B, Schmitt B, Zeidler R, Gires O: The carcinomaassociated antigen EpCAM upregulates c-myc and induces cell proliferation. Oncogene 2004, 23:5748-5758.

4. Osta WA Chen Y, Mikhitarian K, Mitas M, Salem M, Hannun YA Cole DJ, Gillanders WE: EpCAM is overexpressed in breast cancer and is a potential target for breast cancer gene therapy. Cancer Res 2004, 64:5818-5824.

5. Maetzel D, Denzel S, Mack B, Canis M, Went $P$, Benk M, Kieu C, Papior $P$, Baeuerle PA, Munz M, Gires $O$ : Nuclear signalling by tumour-associated antigen EpCAM. Nat Cell Biol 2009, 11:162-171.

6. Carpenter G, Red Brewer M: EpCAM: another surface-to-nucleus missile. Cancer Cell 2009, 15:165-166.

7. Sankpal NV, Willman MW, Fleming TP, Mayfield JD, Gillanders WE: Transcriptional repression of epithelial cell adhesion molecule contributes to p53 control of breast cancer invasion. Cancer Res 2009 69:753-757.

8. Spizzo G, Went $P$, Dirnhofer $S$, Obrist $P$, Simon $R$, Spichtin $H$, Maurer $R$ Metzger U, von Castelberg B, Bart R, Stopatschinskaya S, Köchli OR, Haas P, Mross F, Zuber M, Dietrich H, Bischoff S, Mirlacher M, Sauter G, Gastl G: High Ep-CAM expression is associated with poor prognosis in nodepositive breast cancer. Breast Cancer Res Treat 2004, 86:207-213.

9. Went PT, Lugli A, Meier S, Bundi M, Mirlacher M, Sauter G, Dirnhofer S: Frequent EpCam protein expression in human carcinomas. Hum Pathol 2004, 35:122-128.

10. Herlyn M, Steplewski Z, Herlyn D, Koprowski H: Colorectal carcinomaspecific antigen: detection by means of monoclonal antibodies. Proc Natl Acad Sci USA 1979, 76:1438-1442.

11. Sears HF, Atkinson B, Mattis J, Ernst C, Herlyn D, Steplewski Z, Hayry P, Koprowski H: Phase-I clinical trial of monoclonal antibody in treatment of gastrointestinal tumours. Lancet 1982, 1:762-765.

12. Naundorf S, Preithner S, Mayer P, Lippold S, Wolf A, Hanakam F, Fichtner I, Kufer $P$, Raum T, Riethmüller $G$, Baeuerle PA, Dreier $T$ : In vitro and in vivo activity of MT201, a fully human monoclonal antibody for pancarcinoma treatment. Int J Cancer 2002, 100:101-110.

13. Prang N, Preithner S, Brischwein K, Goster P, Woppel A, Muller J, Steiger C Peters M, Baeuerle PA, da Silva AJ: Cellular and complement-dependent cytotoxicity of Ep-CAM-specific monoclonal antibody MT201 against breast cancer cell lines. Br J Cancer 2005, 92:342-349.
14. Velders MP, Litvinov SV, Warnaar SO, Gorter A, Fleuren GJ, Zurawski VR Jr, Coney LR: New chimeric anti-pancarcinoma monoclonal antibody with superior cytotoxicity-mediating potency. Cancer Res 1994, 54:1753-1759.

15. Velders MP, van Rhijn CM, Briaire $\mathbb{H}$, Fleuren GJ, Warnaar SO, Litvinov SV: Immunotherapy with low and high affinity monoclonal antibodies 17-1A and 323/A3 in a nude mouse xenograft carcinoma model. Cancer Res 1995, 55:4398-4403.

16. Ammons WS, Bauer RJ, Horwitz AH, Chen ZJ, Bautista E, Ruan HH, Abramova M, Scott KR, Dedrick RL: In vitro and in vivo pharmacology and pharmacokinetics of a human engineered monoclonal antibody to epithelial cell adhesion molecule. Neoplasia 2003, 5:146-154.

17. de Bono JS, Tolcher AW, Forero A, Vanhove GF, Takimoto C, Bauer RJ Hammond LA, Patnaik A, White ML, Shen S, Khazaeli MB, Rowinsky EK LoBuglio AF: ING-1, a monoclonal antibody targeting Ep-CAM in patients with advanced adenocarcinomas. Clin Cancer Res 2004, 10:7555-7565.

18. Spizzo G, Obrist P, Ensinger C, Theurl I, Dunser M, Ramoni A, Gunsilius E, Eibl G, Mikuz G, Gastl G: Prognostic significance of Ep-CAM AND Her-2/ neu overexpression in invasive breast cancer. Int J Cancer 2002, 98:883-888.

19. Schmidt M, Hasenclever D, Schaeffer M, Boehm D, Cotarelo C, Steiner E, Lebrecht A, Siggelkow W, Weikel W, Schiffer-Petry I, Gebhard S, Pilch H, Gehrmann M, Lehr HA, Koelbl H, Hengstler JG, Schuler M: Prognostic effect of epithelial cell adhesion molecule overexpression in untreated nodenegative breast cancer. Clin Cancer Res 2008, 14:5849-5855

20. Cimino A, Halushka M, Illei P, Wu X, Sukumar S, Argani P: Epithelial cell adhesion molecule (EpCAM) is overexpressed in breast cancer metastases. Breast Cancer Res Treat 2010, 123:701-708.

21. Went $P$, Vasei $M$, Bubendorf $L$, Terracciano L, Tornillo $L$, Riede $U$, Kononen J, Simon R, Sauter G, Baeuerle PA: Frequent high-level expression of the immunotherapeutic target Ep-CAM in colon, stomach, prostate and lung cancers. Br J Cancer 2006, 94:128-135.

22. Kimura $H$, Kato $H$, Faried $A$, Sohda M, Nakajima M, Fukai $Y$, Miyazaki $T$, Masuda N, Fukuchi M, Kuwano H: Prognostic significance of EpCAM expression in human esophageal cancer. Int J Oncol 2007, 30:171-179.

23. Songun I, Litvinov SV, van de Velde CJ, Pals ST, Hermans J, van Krieken JH: Loss of Ep-CAM (CO17-1A) expression predicts survival in patients with gastric cancer. $\mathrm{Br} J$ Cancer 2005, 92:1767-1772

24. Seligson DB, Pantuck AJ, Liu X, Huang Y, Horvath S, Bui MH, Han KR, Correa AJ, Eeva M, Tze S, Belldegrun AS, Figlin RA: Epithelial cell adhesion molecule (KSA) expression: pathobiology and its role as an independent predictor of survival in renal cell carcinoma. Clin Cancer Res 2004, 10:2659-2669.

25. Went $P$, Dirnhofer $S$, Salvisberg $T$, Amin MB, Lim SD, Diener PA, Moch $H$ : Expression of epithelial cell adhesion molecule (EpCam) in renal epithelial tumors. Am J Surg Pathol 2005, 29:83-88.

26. Spizzo G, Went $P$, Dirnhofer S, Obrist $P$, Moch H, Baeuerle PA, MuellerHolzner E, Marth C, Gastl G, Zeimet AG: Overexpression of epithelial cell adhesion molecule (Ep-CAM) is an independent prognostic marker for reduced survival of patients with epithelial ovarian cancer. Gynecol Oncol 2006, 103:483-488

27. Varga M, Obrist P, Schneeberger S, Mühlmann G, Felgel-Farnholz C, Fong D, Zitt M, Brunhuber T, Schäfer G, Gastl G, Spizzo G: Overexpression of epithelial cell adhesion molecule antigen in gallbladder carcinoma is an independent marker for poor survival. Clin Cancer Res 2004, 10:3131-3136.

28. Fong D, Steurer M, Obrist P, Barbieri V, Margreiter R, Amberger A, Laimer K, Gastl G, Tzankov A, Spizzo G: Ep-CAM expression in pancreatic and ampullary carcinomas: frequency and prognostic relevance. J Clin Pathol 2008, 61:31-35

29. Basak S, Speicher D, Eck S, Wunner W, Maul G, Simmons MS, Herlyn D: Colorectal carcinoma invasion inhibition by C017-1A/GA733 antigen and its murine homologue. J Natl Cancer Inst 1998, 90:691-697.

30. Tai KY, Shiah SG, Shieh YS, Kao YR, Chi CY, Huang E, Lee HS, Chang LC, Yang PC, Wu CW: DNA methylation and histone modification regulate silencing of epithelial cell adhesion molecule for tumor invasion and progression. Oncogene 2007, 26:3989-3997.

31. Ventura A, Meissner A, Dillon CP, McManus M, Sharp PA, Van Parijs L, Jaenisch R, Jacks T: Cre-lox-regulated conditional RNA interference from transgenes. Proc Natl Acad Sci USA 2004, 101:10380-10385.

32. Sankpal NV, Fleming TP, Gillanders WE: Dual expression lentiviral vectors for concurrent RNA interference and rescue. J Surg Res 2009, 156:50-56. 
33. Herr I, Krilleke D, Debatin KM: A sensitive, non-radioactive and fast method for detection of JNK/SAPK activity in leukemic T cells. Leukemia 2000, 14:1859-1860.

34. Dawson PJ, Wolman SR, Tait L, Heppner GH, Miller FR: MCF10AT: a model for the evolution of cancer from proliferative breast disease. Am J Pathol 1996, 148:313-319.

35. Heppner GH, Miller FR, Shekhar PM: Nontransgenic models of breast cancer. Breast Cancer Res 2000, 2:331-334.

36. Santner SJ, Dawson PJ, Tait L, Soule HD, Eliason J, Mohamed AN, Wolman SR, Heppner GH, Miller FR: Malignant MCF10CA1 cell lines derived from premalignant human breast epithelial MCF10AT cells. Breast Cancer Res Treat 2001, 65:101-110.

37. Denzel S, Maetzel D, Mack B, Eggert C, Barr G, Gires O: Initial activation of EpCAM cleavage via cell-to-cell contact. BMC Cancer 2009, 9:402.

38. Ozanne BW, Spence HJ, McGarry LC, Hennigan RF: Transcription factors control invasion: AP-1 the first among equals. Oncogene 2007, 26:1-10.

39. Eferl R, Wagner EF: AP-1: a double-edged sword in tumorigenesis. Nat Rev Cancer 2003, 3:859-868.

40. Brenner DA, O'Hara M, Angel P, Chojkier M, Karin M: Prolonged activation of jun and collagenase genes by tumour necrosis factor-alpha. Nature 1989, 337:661-663.

41. Westwick JK, Weitzel C, Minden A, Karin M, Brenner DA: Tumor necrosis factor alpha stimulates AP-1 activity through prolonged activation of the c-Jun kinase. The J Biol Chem 1994, 269:26396-26401.

42. Bennett BL, Sasaki DT, Murray BW, O'Leary EC, Sakata ST, Xu W, Leisten JC, Motiwala A, Pierce S, Satoh Y, Bhagwat SS, Manning AM, Anderson DW: SP600125, an anthrapyrazolone inhibitor of Jun N-terminal kinase. Proc Natl Acad Sci USA 2001, 98:13681-13686.

43. Hu YF, Li R: JunB potentiates function of BRCA1 activation domain 1 (AD1) through a coiled-coil-mediated interaction. Genes Dev 2002, 16:1509-1517.

44. Langer $\mathrm{S}$, Singer CF, Hudelist $\mathrm{G}$, Dampier B, Kaserer $\mathrm{K}$, Vinatzer $U$, Pehamberger $\mathrm{H}$, Zielinski C, Kubista E, Schreibner M: Jun and Fos family protein expression in human breast cancer: correlation of protein expression and clinicopathological parameters. Eur J Gynaecol Oncol 2006 27:345-352.

45. Vleugel MM, Greijer AE, Bos R, van der Wall E, van Diest PJ: c-Jun activation is associated with proliferation and angiogenesis in invasive breast cancer. Hum Pathol 2006, 37:668-674.

46. Liu Y, Lu C, Shen Q, Munoz-Medellin D, Kim H, Brown PH: AP-1 blockade in breast cancer cells causes cell cycle arrest by suppressing $\mathrm{G} 1$ cyclin expression and reducing cyclin-dependent kinase activity. Oncogene 2004, 23:8238-8246.

47. Lu C, Shen Q, DuPre E, Kim H, Hilsenbeck S, Brown PH: cFos is critical for MCF-7 breast cancer cell growth. Oncogene 2005, 24:6516-6524.

48. Shen Q, Uray IP, Li Y, Krisko TI, Strecker TE, Kim HT, Brown PH: The AP-1 transcription factor regulates breast cancer cell growth via cyclins and E2F factors. Oncogene 2008, 27:366-377.

49. Wang J, Kuiatse I, Lee AV, Pan J, Giuliano A, Cui X: Sustained c-Jun-NH2kinase activity promotes epithelial-mesenchymal transition, invasion, and survival of breast cancer cells by regulating extracellular signalregulated kinase activation. Mol Cancer Res 2010, 8:266-277.

50. Jiao X, Katiyar S, Willmarth NE, Liu M, Ma X, Flomenberg N, Lisanti MP Pestell RG: c-Jun induces mammary epithelial cellular invasion and breast cancer stem cell expansion. J Biol Chem 2010, 285:8218-8226.

51. Gostner JM, Fong D, Wrulich OA, Lehne F, Zitt M, Hermann M, Krobitsch S, Martowicz A, Gastl G, Spizzo G: Effects of EpCAM overexpression on human breast cancer cell lines. BMC Cancer 2011, 11:45.

52. Morlon A, Sassone-Corsi P: The LIM-only protein FHL2 is a seruminducible transcriptional coactivator of AP-1. Proc Natl Acad Sci USA 2003, 100:3977-3982

53. Maaser K, Borlak J: A genome-wide expression analysis identifies a network of EpCAM-induced cell cycle regulators. Br J Cancer 2008, 99:1635-1643.

doi: $10.1186 / \mathrm{bcr} 3070$

Cite this article as: Sankpal et al:: Activator protein 1 (AP-1) contributes to EpCAM-dependent breast cancer invasion. Breast Cancer Research 2011 13:R124.

\section{Submit your next manuscript to BioMed Central and take full advantage of:}

- Convenient online submission

- Thorough peer review

- No space constraints or color figure charges

- Immediate publication on acceptance

- Inclusion in PubMed, CAS, Scopus and Google Scholar

- Research which is freely available for redistribution

Submit your manuscript at www.biomedcentral.com/submit 